\title{
Entrainment of Luminiferous Medium with Massive Solids
}

\author{
Alexander I. Korolev \\ St. Petersburg, Russia \\ Email: alex-korolev@ya.ru
}

How to cite this paper: Korolev, A.I. (2017) Entrainment of Luminiferous Medium with Massive Solids. Open Access Library Journal, 4: e4051.

https://doi.org/10.4236/oalib.1104051

Received: October 21, 2017

Accepted: November 21, 2017

Published: November 24, 2017

Copyright ( 2017 by author and Open Access Library Inc.

This work is licensed under the Creative Commons Attribution International License (CC BY 4.0).

http://creativecommons.org/licenses/by/4.0/

\section{(c) () Open Access}

\begin{abstract}
Experiments on searching the space flows of luminiferous medium through Sagnac interferometer have been conducted. To do this, in one of the interferometer arms the inhomogeneity has been introduced in the form of a transparent tube with distilled water. The difference in speeds of the interferometer was registered in relation to the medium when changing the tube orientation from S-N to W-E: By shifting the interference fringe on the screen. Measurements were carried out in two places: 30.2 East longitude/60.5 North latitude at a height of $165 \mathrm{~m}$ above sea level; 41.3 East longitude/43.6 North latitude at height $2850 \mathrm{~m}$. Flows with speeds $>127 \mathrm{~m} / \mathrm{s}$ were not observed. The result is explained by entrainment of the medium due to gravitational attraction to the Earth, the Sun and Center of the Milky Way: Similar to how it happens to other physical medium.
\end{abstract}

\section{Subject Areas}

Modern Physics

\section{Keywords}

Luminiferous Medium, Aether, Dark Matter, WIMP

\section{Introduction}

The search history of the luminiferous medium (world aether) movements begins from the mid- $19^{\text {th }}$ century and continues until now [1]-[11] by independent researchers. Positive results were obtained from the experiments of D. Miller: On the airship, and in the Mount Wilson Observatory (altitude of 1742 meters). After the experimental data was processed, it was concluded that there was an ether wind at a speed of $10-11 \mathrm{~km} / \mathrm{s}$, blowing from apex in the Draco constellation [7]. The negative results of other researchers can be explained by the shiel- 
ding of aether flows with metal casings of interferometers and atmosphere near the Earth's surface. However, this explanation is not consistent with the hypothesis of high penetration capacity of world aether.

Significance of the fundamental term "luminiferous aether" in modern physics is described in [11] [12]. In the work [13] the installation for searching flows of luminiferous medium with speeds $>20 \mathrm{~km} / \mathrm{s}$ at low altitude was used. They were not found. One can use the interferometry [14] methods to register the smaller speeds of movement relative to luminiferous medium. The methods of measuring linear velocity with Mach-Zender interferometer [15] and Sagnac interferometer [16] are known. The latter is more effective because interfering beams pass through the same optical path. This reduces the error due to deformations and increases the efficiency 2 times, because two rays pass through the arm with inhomogeneity (in opposite directions). In this work, we search for the medium flows according to the method [16] with the difference in speed projections to the directions of S-N and W-E $>127 \mathrm{~m} / \mathrm{s}$. Measurements were performed at high altitudes using an open interferometer.

\section{Description of Experiments}

Figure 1 provides a scheme of the experimental installation. On the wooden bar with length of $1.15 \mathrm{~m} \mathrm{1}$ ) laser module is fixed 2). At the module output there is a diaphragm of $5 \mathrm{~mm}$ in diameter. The module is actuated after application of 3 VDC from two batteries. Collimated beam of radiation appears on the wavelength of $635 \mathrm{~nm}$. Along the beam edges in the cross-section there are interference rings due to diffraction on the diaphragm. The beam is splitted with a beam-splitting cube 3) into two parts equal in intensity. Each part passes the same optical path, formed by reflective prisms 4 ), in different directions. In the long arm of the interferometer, there is an acrylic tube with distilled water inside 5). Transparent view glass windows are glued to the ends of the tube. The tube length is $1 \mathrm{~m}$. A partially transparent screen of black tissue paper 6) is located at the interferometer exit. On the back of the screen, beam images are observed in the form of bright red spots. There are also less bright spots due to Fresnel reflection in interferometer elements that do not interfere with the observation of major spots. For registration of the resulting images, the camera operating in close-up photography mode 7) is used.

Before starting the measurements, the tube was refilled with water through the

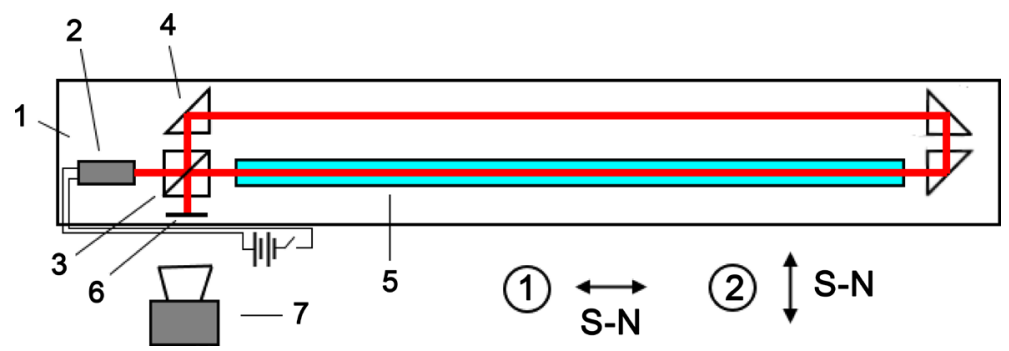

Figure 1. The plant layout. 
side opening using a syringe. This was done to remove the bubbles having been formed during the installation transportation. The adjustment of optical system was then carried out with the purpose of partial matching of two beams images on the screen and the interference pattern observation. Alignment was carried out by rotating the prism secured on the bar with plasticine. On the screen, the interference pattern was observed in the form of alternating light and dark fringes.

If the velocity component of the installation motion relative to luminiferous medium along the direction of S-N differs from that one in the direction of W-E then with appropriate orientations of the tube with water, the interference patterns which differ by the fringes displacement should be seen. To detect this shift, the installation turned in the specified directions many times. Photos of fringes on the screen were processed in the graphics editor and the images of the most visible fringes were compared. For images binding, the opaque mark glued to the screen near the fringes was used.

The first experiment was held on the mountain near Sosnovo town (60.5 North latitude 30.2 East longitude) at a height of $165 \mathrm{~m}$ above sea level. Date and time of shooting: 25.03.2017 from 14:00 to 16:00. The second experiment was held on the Abishir-Ahuba ridge near the village of Arkhyz (43.6 North latitude 41.3 East longitude) at a height of $2850 \pm 50 \mathrm{~m}$. Date and Time: 25.07.2017 from 7:00 to 8:00. The places for installation were chosen in such a way that Draco constellation was in the zone of direct visibility from which the flow of luminiferous medium was supposed could be registered. The projection of this direction onto the Earth surface is roughly corresponds to the north direction. Additionally, the velocity accuracy measurements allowed recording the medium motion due to rotation of the Earth, Sun and center of the Milky Way. Before measurements the protective cover was removed from interferometer. In experiment No. 1, the installation was placed on tripod under the plastic tent, in Experiment No. 2-on the vibration-insulating flat in the tent with a polyether roof.

\section{Results and Discussion}

In each experiment, there were at least 10 successful frames with sharp image of fringes. On Figure 2(a) \& Figure 2(b), pairs of the brightest images of dark fringes on light spots are presented: in the directions of S-N and W-E. The fringes have significant distortions due to non-parallelism of the tube view windows, their roughness, presence of impurities in the water and possible contamination on the prism surfaces after alignment. The difference in the fringes forms between pairs is due to interferometer misalignment. In one couple, there is no such difference because of the little delay between the frames in it. Distortion does not prevent you from exploring the fringes shift. The quality of the images obtained makes it possible to identify it with an accuracy up to $20 \%$ of the distance between adjacent fringes. With such accuracy shift is not observed. 


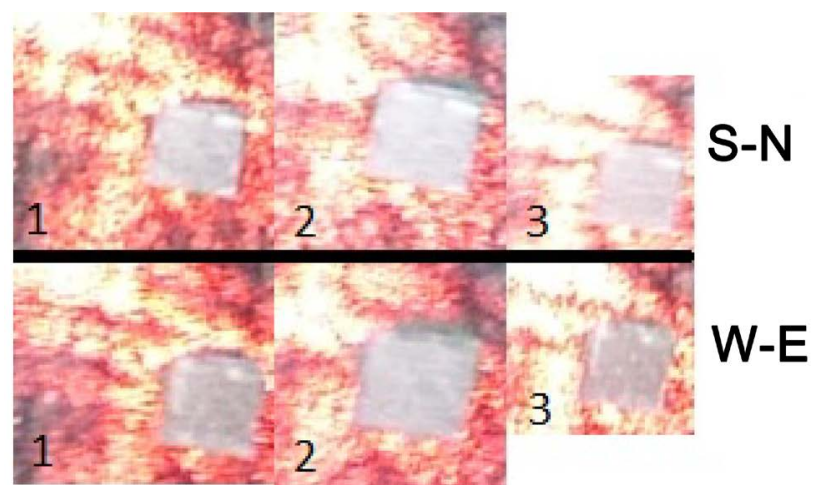

(a)

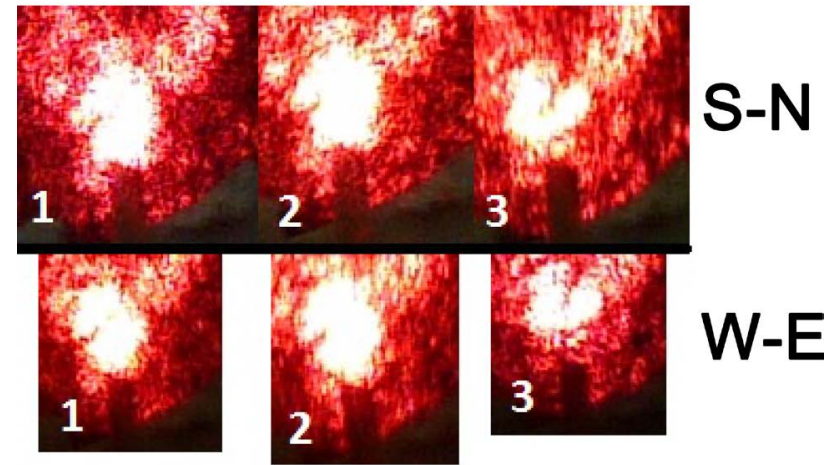

(b)

Figure 2. Pairs of the interference patterns on the screen. (a) In experiment №1; (b) in experiment №2. The marks width is 1 - $2 \mathrm{~mm}$.

Fringes shift can emergence under difference in speed of the laser beams propagating towards each other in the tube with water. This is in the case of water motion relative to the luminiferous medium. This shift value can be determined by the formula (1):

$$
\Delta x=\frac{x}{\lambda} \cdot \delta
$$

Here, $x$ is the distance between adjacent fringes, $\lambda$ is the wavelength, $\delta$ is path difference of the beams caused by the water motion relative to luminiferous medium. $\delta$ is calculated by the formula (2):

$$
\delta=2\left|\Delta c_{w}\right| \cdot t \simeq \frac{2 V L}{c n}
$$

Here $\Delta c_{w}$ is the difference of lightspeed in the water relatively the tube: when moving and stationary, $t$ is the time of light passing through the tube, $n \simeq 1.33$ is the water refraction index, $L$ is the length of the tube, $V$ is the speed of installation movement relative to the luminiferous medium in the direction of tube axis. $\Delta c_{w}$ is obtained from the Fizeau experiment [2]. Thus, $V$ is calculated according to the formula (3):

$$
V \simeq \frac{\Delta x}{x} \cdot \frac{\lambda c n}{2 L}
$$


With no shift, the speed is equal to zero. Taking into account the error in defining the fringes shift and provided that the medium is stationary in the direction perpendicular to the tube axis $\mathrm{V}=0 \pm 127 \mathrm{~m} / \mathrm{s}$. In experiments, the velocity components may have been present in a perpendicular direction because of space motions. However, their difference from the velocity in the main direction must be $>127 \mathrm{~m} / \mathrm{s}$, which is not observed. Thus, the lack of luminiferous medium space motion has been identified with the specified precision.

The result obtained demonstrates that the luminiferous medium is moving around the Earth, the Sun, and the center of the Milky Way synchronously along with installation. Hippolyte Fizeau believed that the luminiferous aether in his experiment was entrained by the flow of fluid or gas [2]. But this explanation is not suitable for air atmosphere with a refracting index close to 1 . Moreover, the full entrainment is observed with a sufficiently high accuracy. The results of the experiments can be explained provided that the luminiferous medium has a mass and viscosity. Mass-in order to be gravitated by celestial bodies, viscosity-in order to be rotated by them. The medium rotation in the experiment is registered by the lack of fringes shift due to the Earth's rotation. The massiveness and viscosity of the luminiferous medium are consistent with some classic concepts of it [17]. In this case, the high penetrating ability provides a weak interaction with a visible matter. In Fizeau's experiment the change of the lightspeed in moving medium can be explained by the difference in the braking action of the medium to photons beam (light) at different speeds of the medium, just as it would be with flow of other massive particles. The difference of the effective (radiation) mass of photon from the medium mass in its volume is described in [18]. These two masses may also be called "inertial" and "gravitational". The inertial mass is responsible for inertial interactions of the waves formations of luminiferous medium-photons (entrainment by moving medium with refraction index $>1$, pressure on a target). Gravitational mass is responsible for gravitational (and viscosity) interactions of the luminiferous medium itself.

In modern physical theory, there is a hypothesis about the existence of Weakly Interacting Massive Particles (WIMP), whose gas is considered to be a candidate for the cold part of dark matter [19]. Assuming that this gas (or medium in another aggregative state) has viscosity and is the medium for electromagnetic waves (light) propagation, the result of this work will be explained in the framework of the modern theory. There will also be a simple explanation of effects such as gravitational lensing and gravitational redshift. The light beam deviation by the massive body when lensing is due to the gravitational attraction of the propagation medium. The redshift in a light beam moving away from the massive body is explained by the medium extension along with the beam due to reduction of gravitational stresses in the medium.

\section{Conclusion}

The search for the linear velocity motion of the luminiferous medium around 
the Earth, the Sun and the center of our Galaxy has led to the zero result with accuracy of $127 \mathrm{~m} / \mathrm{s}$. The measurements were carried out at high altitude with the purpose to reduce the atmosphere and objects impact on the Earth's surface. In order to reduce the probable shielding of the luminiferous medium flows, the installation used interferometer without casing. The result is explained by entrainment of luminiferous medium with gravitating bodies because of its mass and viscosity. The similarity due to probably massivity of this medium elemental parts-particles, usually referred as "amers" [11], with hypothetical particles of dark matter WIMP brings together the classical conceptions of the world aether and the modern physical theory.

\section{References}

[1] Arago, A. (1810-1853) Mémoire sur la vitesse de la lumière, lu à la prémière classe de l'Institut, le 10 décembre 1810. Comptes Rendus de l'Académie des Sciences, 36, 38-49.

[2] Fizeau, H. (1849) Sur une experience relative a la vitesse de propagation de la Lumiere. Compte Rendu, 29, 90-92.

[3] Sagnac, G. (1913) L'ether lumineux demontre par l'effet du vent relatif d'ether dans un interferometre en rotation uniforme. Comptes Rendus, 157, 708-710.

[4] Michelson, A. and Morley, E. (1887) On the Relative Motion of the Earth and the Luminiferous Ether. American Journal of Science, 34, 333-345.

https://doi.org/10.2475/ajs.s3-34.203.333

[5] Michelson, A., Pease, F. and Pearson, F. (1929) Results of Repetition of the Michelson-Morley Experiment. Journal of the Optical Society of America, 18, 181.

[6] Miller, D. (1925) Ether-Drift Experiments at Mount Wilson. Proceedings of the National Academy of Sciences, 11, 306-314.

[7] Miller, D. (1933) The Ether-Drift Experiment and the Determination of the Absolute Motion of the Earth. Reviews of Modern Physics, 5, 203-242.

https://doi.org/10.1103/RevModPhys.5.203

[8] Kennedy, R. (1926) A Refinement of the Michelson-Morley Experiment. Proceedings of the National Academy of Sciences, 12, 621-629.

https://doi.org/10.1073/pnas.12.11.621

[9] Illingworth, K. (1927) A Repetition of the Michelson-Morley Experiment Using Kennedy's Refinement. Physical Review, 30, 692-696. https://doi.org/10.1103/PhysRev.30.692

[10] Joos, G. (1930) Die Jenaer Wiederholung des Michelsonversuchs. Annalen der Physik, 399, 385-407.

[11] Atsyukovsky, V. (1993) Ether Wind. Collection of Papers. Energoatomizdat, Moscow, 172.

[12] Spavieri, G., Guerra, V., de Abreu, R. and Gillies, G. (2008) Ether Drift Experiments and Electromagnetic Momentum. The European Physical Journal D, 47, 457-463. https://doi.org/10.1140/epjd/e2008-00062-x

[13] Korolev, A. (2014) A Method of Luminiferous Ether Registration. Open Access Library, 1, e125. https://doi.org/10.4236/oalib.preprints.1200125

[14] Wolter, K. (2007) Speed Measurement. Patent US 2007/0186653 A1.

[15] Belousov, A., et al. (2005) Device for Measuring Speed of Motion of Transportation 
Vehicle. Patent RU 2261449 C2.

[16] Jiajun, Z. (2015) Speed Measurement Method and Device Adopting Light Ray Interferometer. Patent CN 104655870 A.

[17] Burago, S. (2017) The Natural Explanation of Some of the Properties of Light, Which Are Used to Confirm the Theory Einstein's Relativity. http://gsjournal.net/Science-Journals/Research\%20Papers/View/6832

[18] Korolev, A. (2017) On the Nature of Photon. Open Access Library, 3, e162. https://doi.org/10.4236/oalib.preprints.1200162

[19] Kamionkowski, M. (1997) WIMP and Axion Dark Matter. arXiv:hep-ph/9710467

Submit or recommend next manuscript to OALib Journal and we will provide best service for you:

- Publication frequency: Monthly

- 9 subject areas of science, technology and medicine

- Fair and rigorous peer-review system

- Fast publication process

- Article promotion in various social networking sites (LinkedIn, Facebook, Twitter, etc.)

- Maximum dissemination of your research work

Submit Your Paper Online: Click Here to Submit

Or Contact service@oalib.com 